\title{
Nonlinear Adaptive Partial State Feedback Trajectory Tracking Control of Tendon Driven Robot Manipulators
}

\author{
Beytullah Okur, Erkan Zergeroglu, Enver Tatlicioglu, Orhan Aksoy
}

\begin{abstract}
In this work, the link position tracking control problem of a tendon driven robotic system is studied in the presence of parametric uncertainty and lack of velocity measurements both of links and actuators. A partial state feedback nonlinear adaptive controller is proposed to deal with the unmeasurable states and uncertain dynamical system parameters. A backstepping approach has been utilized to develop the control strategy. The proposed nonlinear tracking controller utilizes online update laws to adapt for parametric uncertainties, and requires only link and actuator position measurements and tendon tension measurements. Need for link velocity measurements are eliminated by using a nonlinear filter, and a set of linear filters is designed to estimate the actuator velocities. Lyapunov based arguments have been applied to prove the stability of the closed-loop system and semi-global asymptotic link position tracking is achieved.
\end{abstract}

\section{INTRODUCTION}

Separating the actuators from the links of the robot and actuating each joint remotely would decrease the link size, mass and inertia of the robot manipulator. As this might become a key point in light weighted, agile robot design, a significant amount of research has focused on remotely actuating robotic systems. Among other remote power transmitting methods, the use of tendon transmission systems present less noisy, clean, and shock absorbent characteristics. Therefore, tendon driven mechanisms have been used in many robotic applications. To name some; [1], [2], [3] can be given as examples to small size applications such as robotic hands, and [4], [5], [6] are some examples for large size manipulators. The use of tendon driven actuation is more popular in dexterous hands [2], [3], [7] as the resultant task space motion in robotic hand design usuually does not need to be accurate. For applications where the main performance criteria is to accurately track a desired task space trajectory, the use of tendon driven mechanisms are limited which is mostly due to the elastic nature of the tendons where accurate position control and trajectory tracking becomes difficult. And in most model based controllers, it is necessary to include the elastic tendon dynamics to the system model, however, with this inclusion, the control problem becomes

This research is supported by Grants of the Scientific and Technological Research Council of Turkey, TUBITAK Project No: 112E561.

B. Okur is with the Department of Mechatronic Engineering, Yildiz Tech. University, Besiktas, Istanbul, Turkey.

okur@yildiz.edu.tr

E. Zergeroglu and O. Aksoy are with the Department of Computer Engineering, Gebze Institute of Technology, 41400, Gebze, Kocaeli, Turkey

ezerger@bilmuh.gyte.edu.tr

E. Tatlicioglu is with the department of Electrical and Electronics Engineering, Izmir Institute of Technology, Izmir, 35430 Turkey

enveraiyte.edu.tr more complicated due to the extra dynamics and hence possible extra uncertainties [5].

Some part of past research has focused on designing controller for tendon driven systems. For some background on tendon driven robot manipulators and classical linear control approaches on tendon driven systems, the reader is referred to [8], [9], and the references therein. Controller formulations including system dynamics are limited in the literature. In [5], Kobayashi and Ozawa presented an adaptive neural network based controller for tendon driven robotic mechanisms with elastic tendons. In [10], Nakayama and Fujimoto tackled the tracking control of tendon driven robots by applying the delayed reflexive force feedback. In [11] and [12], Haiya et al. proposed controllers for multiple degreeof-freedom (dof) tendon mechanisms using nonlinear springs with hysteresis characteristics like stiffness adjustable tendons. For the proposed controllers, error of the equation of spring was estimated by a disturbance observer and compensated by utilizing the estimated disturbance. In [13], Wimbock et al. proposed an application of the Immersion and Invariance type framework to tendon driven systems with variable stiffness. Among the above cited work, the only work that considered the uncertainties in the system dynamics was given in [5], however the proposed adaptive controller required the measurement of the second and third time derivatives of link position measurements (see assumption (2) of [5]) which are usually not available.

In this study, we design a nonlinear model based partial state feedback adaptive controller for tendon driven robot manipulators that does not require neither acceleration measurements nor velocity measurements. The proposed controller only requires link and actuator position measurements and tension measurements of each tendons. Specifically, the proposed semi-global adaptive partial state feedback trajectory tracking controller deals with parametric uncertainties via three different parameter update rules. The need for link velocity measurements are eliminated by utilizing a nonlinear link velocity filter during the error system development, and the lack of actuator velocity measurements have been overcome with the help of a set of linear filters. Adding the dynamics of the power transmission system and considering tendon elasticity yield a complicated dynamic model, and the resulting system dynamics mandates the use the backstepping technique twice. After fusing the backstepping design procedure with Lyapunov-type analysis tools, we design the auxiliary backstepping control inputs, and the control input applied to the actuators. The stability analysis ensures boundedness of all the signals under the closed-loop 
operation, and semi-global asymptotic tracking of the link position error.

The rest of this work is organized in the following manner. The dynamical model of tendon driven manipulators along with the controller formulation is presented in Section II. Stability analysis of the closed-loop system is given in Section III. Concluding remarks are given in Section IV.

\section{SySTEM DYNAMICS AND CONTROLLER FORMULATION}

The dynamics of $n$-dof robot manipulator driven by an $m$-tendon mechanism is considered to have the following form [5]

$$
\begin{gathered}
M(q) \ddot{q}+V_{m}(q, \dot{q}) \dot{q}+F_{d} \dot{q}+G(q)=-J_{j}^{T}(q) f_{t}(l) \\
J \ddot{\theta}+B \dot{\theta}+R_{a} f_{t}(l)=\tau_{a} \\
\dot{l}=J_{j}(q) \dot{q}+R_{a} \dot{\theta}
\end{gathered}
$$

where $q(t), \dot{q}(t), \ddot{q}(t) \in \mathbb{R}^{n}$ represent the link position, velocity and acceleration vectors, respectively, $\theta(t), \dot{\theta}(t)$, $\ddot{\theta}(t) \in \mathbb{R}^{m}$ represents the actuator position, velocity and acceleration vectors, respectively, $l(t), i(t) \in \mathbb{R}^{m}$ are the tendon expansion vector and its time derivative, $M(q) \in$ $\mathbb{R}^{n \times n}$ denotes the link inertia effects, $V_{m}(q, \dot{q}) \in \mathbb{R}^{n \times n}$ represents the centripetal-Coriolis effects, $G(q) \in \mathbb{R}^{n}$ denotes the gravitational terms related to the robot, $F_{d} \in \mathbb{R}^{n \times n}$ is the constant diagonal link viscous friction matrix, $J$, $B \in \mathbb{R}^{m \times m}$ are the diagonal actuator inertia and viscous friction matrices, respectively, $R_{a} \in \mathbb{R}^{m \times m}$ is the diagonal matrix containing the radius of pulleys mounted on each actuator, $J_{j}(q) \in \mathbb{R}^{m \times n}$ is the Jacobian matrix that maps the joint space to the tendon expansion space, $f_{t}(l) \in \mathbb{R}^{m}$ is the vector of tendon tensile forces generated by the tendon expansions, and finally $\tau_{a}(t) \in \mathbb{R}^{m}$ is the control input vector applied the actuators.

The control objective is to design a link position tracking controller for the tendon driven robot manipulator model given by (1)-(3) under the restrictive constraint that the dynamical system parameters of (1) are uncertain, and velocity measurements of both robot links and actuators are not available. Specifically, the controller should ensure the robot links to asymptotically follow a desired trajectory despite the uncertain robot model parameters, and lack of link and actuator velocity measurements.

In order to quantify the tracking control objective, we define the link position tracking error $e(t) \in \mathbb{R}^{n}$ as follows

$$
e \triangleq q_{d}-q
$$

where it is assumed that the desired link position signal $q_{d}(t)$ and its time derivatives are sufficiently smooth and bounded functions of time. We define a filtered tracking error like term $\eta(t) \in \mathbb{R}^{n}$ as follows

$$
\eta \triangleq \dot{e}+\alpha_{1} e+\alpha_{2} e_{f}
$$

where $\alpha_{1}, \alpha_{2} \in \mathbb{R}$ are positive constant filter gains, and $e_{f}(t) \in \mathbb{R}^{n}$ is an auxiliary filter variable having the following dynamic expression

$$
\dot{e}_{f}=-\alpha_{3} e_{f}+\alpha_{2} e-k \eta, e_{f}(0)=0_{m \times 1}
$$

where $\alpha_{3} \in \mathbb{R}$ is a positive constant filter gain and $k \in \mathbb{R}$ is a positive constant control gain.

After taking the time derivative of (5), pre-multiplying both sides by $M(q)$, substituting (1), (5) and (6), adding and subtracting $Y_{d} \phi_{r}$ and $V_{m}(q, \dot{q}) \eta$ to the right hand-side of the resulting equation, we can obtain the following expression

$M(q) \dot{\eta}=-V_{m}(q, \dot{q}) \eta+Y_{d} \phi_{r}-\alpha_{2} k M(q) \eta+J_{j}^{T}(q) f_{t}(l)+\chi$

where $\chi(t) \in \mathbb{R}^{n}$ is an auxiliary signal defined as

$$
\begin{aligned}
\chi \triangleq & M(q) \ddot{q}_{d}+V_{m}\left(q, \dot{q}_{d}\right) \dot{q}_{d}+F_{d} \dot{q}+G(q) \\
& -Y_{d} \phi_{r}+\alpha_{1} M(q)\left(\eta-\alpha_{1} e-\alpha_{2} e_{f}\right) \\
& +\alpha_{2} M(q)\left(-\alpha_{3} e_{f}+\alpha_{2} e\right) \\
& -V_{m}(q, \eta)\left(\dot{q}_{d}+\alpha_{1} e+\alpha_{2} e_{f}\right) \\
& +V_{m}\left(q, \dot{q}_{d}\right)\left(\alpha_{1} e+\alpha_{2} e_{f}\right) \\
& +V_{m}\left(q, \dot{q}_{d}+\alpha_{1} e+\alpha_{2} e_{f}\right)\left(\alpha_{1} e+\alpha_{2} e_{f}\right) .
\end{aligned}
$$

At this stage we would like to point out that

$$
\|\chi\| \leq \rho(\|x\|)\|x\|
$$

where $\rho(\cdot)$ is a positive scalar bounding function, and $x(t) \in$ $\mathbb{R}^{3 n}$ is defined as follows

$$
x \triangleq\left[\begin{array}{lll}
e^{T} & e_{f}^{T} & \eta^{T}
\end{array}\right]^{T} .
$$

In order to initiate the first backstepping procedure, we introduce an auxiliary error term, denoted by $\eta_{f}(t) \in \mathbb{R}^{m}$, to reach the tendon dynamics, which is defined as

$$
\eta_{f} \triangleq f_{t}(l)-f_{d}
$$

where $f_{d}(t) \in \mathbb{R}^{m}$ is an auxiliary control input. After adding and subtracting $J_{j}^{T}(q) f_{d}(t)$ to the right-hand side of (7), we obtain the following

$$
\begin{aligned}
M(q) \dot{\eta}= & -V_{m}(q, \dot{q}) \eta+Y_{d} \phi_{r}-\alpha_{2} k M(q) \eta \\
& +\chi+J_{j}^{T}(q) \eta_{f}+J_{j}^{T}(q) f_{d} .
\end{aligned}
$$

Based on the open-loop robot dynamics given in (12), and motivated by the subsequent stability analysis, we design the auxiliary control input $f_{d}(t)$ as

$$
f_{d}=\left[J_{j}^{T}(q)\right]^{+}\left\{-Y_{d} \hat{\phi}_{r}+k K_{s} e_{f}-K_{s} e\right\}
$$

where $K_{s} \in \mathbb{R}$ is a positive scalar gain, $\left[J_{j}^{T}(q)\right]^{+} \triangleq$ $J_{j}(q)\left[J_{j}^{T}(q) J_{j}(q)\right]^{-1} \in \mathbb{R}^{m \times n}$ is the pseudo inverse of $J_{j}^{T}(q)$, and $\hat{\phi}_{r}(t) \in \mathbb{R}^{p}$ is the estimate of $\phi_{r}$ updated via the following

$$
\dot{\hat{\phi}}_{r}=\Gamma Y_{d}^{T} \eta
$$

where $\Gamma \in \mathbb{R}^{p \times p}$ is a positive definite, constant, diagonal adaptation gain matrix. From (14), it is clear that the righthand side depends on $\eta(t)$ which is not available. Subsequently, we will provide an implementable (i.e., link velocity independent) form. The control gain $k$ is chosen as

$$
k=\frac{1}{m_{1}}\left[1+k_{n 1} \rho^{2}(\|x\|)\right]
$$


with $k_{n 1}$ being a positive constant nonlinear damping gain. Substituting (13) into (12) yields the closed-loop dynamics to be obtained as follows

$$
\begin{aligned}
M(q) \dot{\eta}= & -V_{m}(q, \dot{q}) \eta+Y_{d} \tilde{\phi}_{r}-\alpha_{2} k M(q) \eta \\
& +\chi+J_{j}^{T}(q) \eta_{f}+k K_{s} e_{f}-K_{s} e
\end{aligned}
$$

where $\tilde{\phi}_{r}(t) \in \mathbb{R}^{p}$ is the parameter estimation error and defined as

$$
\tilde{\phi}_{r} \triangleq \phi_{r}-\hat{\phi}_{r}
$$

Remark 1: Note that, although the control input in (13) depends on $e_{f}(t)$ (which obviously requires link velocity measurements), we can construct an implementable (i.e., link velocity independent) form of the auxiliary control input $f_{d}(t)$. To construct the link velocity independent version of the filter given by (6), we insert (5) into (6) to reach the following expression

$$
\dot{e}_{f}=-k \dot{e}+\left(\alpha_{2}-k \alpha_{1}\right) e-\left(\alpha_{3}+k \alpha_{2}\right) e_{f}
$$

We now define, an auxiliary signal, denoted by $w(t) \in \mathbb{R}^{n}$, as

$$
w \triangleq e_{f}+k e
$$

whose time derivative can be obtained as

$$
\dot{w}=\left(\alpha_{2}-k \alpha_{1}\right) e-\left(\alpha_{3}+k \alpha_{2}\right)(w-k e)
$$

where (18) and (19) were utilized. From (20), it is clear that $w(t)$ can be obtained from link position measurements only, and thus from (19), one can obtain an implementable form of $e_{f}(t)$. After this, the implementable form of (14) is given as

$$
\begin{aligned}
\hat{\phi}_{r}= & \Gamma \int_{0}^{t} Y_{d}^{T}(\sigma)\left(\alpha_{1} e(\sigma)+\alpha_{2} e_{f}(\sigma)\right) d \sigma \\
& +\Gamma Y_{d}^{T}(t) e(t)-\Gamma Y_{d}^{T}(0) e(0) \\
& +\Gamma \int_{0}^{t} \dot{Y}_{d}^{T}(\sigma) e(\sigma) d \sigma .
\end{aligned}
$$

The control problem was further complicated by the unavailability of the actuator velocity information. To overcome the lack of the actuator velocity measurements, we present the development of a set of linear filters. From the actuator dynamics given in (2), we obtain the following expression

$$
\ddot{\theta}=J^{-1}\left[\tau_{a}-B \dot{\theta}-R_{a} f_{t}(l)\right] .
$$

To write the actuator dynamics in a state space form, we define a new vector $r(t) \in \mathbb{R}^{2 m}$ and auxiliary state variables $r_{1}(t), r_{2}(t) \in \mathbb{R}^{m}$ as follows

$$
r=\left[\begin{array}{l}
r_{1} \\
r_{2}
\end{array}\right] \triangleq\left[\begin{array}{c}
\theta \\
\dot{r}_{1}+J^{-1} B r_{1}
\end{array}\right]
$$

Taking the time derivative of $r_{2}(t)$ and then substituting (22) and (23), we obtain

$$
\dot{r}_{2} \triangleq J^{-1} \tau_{a}-J^{-1} R_{a} f_{t}(l)
$$

The actuator dynamics can be written in the following state space form

$\dot{r}=A_{0} r+\left[\begin{array}{c}k_{f_{1}} I_{m} \\ k_{f_{2}} I_{m}\end{array}\right] r_{1}+A_{1}\left[\begin{array}{c}r_{1} \\ 0_{m \times 1}\end{array}\right]+A_{2}\left[\begin{array}{c}0_{m \times 1} \\ f_{t}(l)\end{array}\right]+\left[\begin{array}{c}0_{m \times 1} \\ J^{-1} \tau_{a}\end{array}\right]$

where $k_{f_{1}}, k_{f_{2}} \in \mathbb{R}$ are constant positive filter gains, $A_{0}$, $A_{1}, A_{2} \in \mathbb{R}^{m \times m}$ are Hurwitz matrices, and are defined as [17]

$$
\begin{aligned}
& A_{0} \triangleq\left[\begin{array}{cc}
-k_{f_{1}} I_{m} & I_{m} \\
-k_{f_{2}} I_{m} & 0_{m \times m}
\end{array}\right] \\
& A_{1} \triangleq\left[\begin{array}{cc}
-J^{-1} B & 0_{m \times m} \\
0_{m \times m} & -J^{-1} B
\end{array}\right] \\
& A_{2} \triangleq\left[\begin{array}{cc}
-J^{-1} R_{a} & 0_{m \times m} \\
0_{m \times m} & -J^{-1} R_{a}
\end{array}\right] .
\end{aligned}
$$

Based on the above dynamics, the following linear, actuator velocity independent filters are designed [18], [19]

$$
\begin{aligned}
& \dot{\varepsilon}_{0}=A_{0} \varepsilon_{0}+\left[\begin{array}{l}
k_{f_{1}} I_{m} \\
k_{f_{2}} I_{m}
\end{array}\right] r_{1} \quad \varepsilon_{0} \triangleq\left[\begin{array}{ll}
\varepsilon_{01}^{T} & \varepsilon_{02}^{T}
\end{array}\right]^{T} \\
& \dot{\varepsilon}_{1}=A_{0} \varepsilon_{1}+\left[\begin{array}{c}
r_{1} \\
0_{m \times 1}
\end{array}\right] \quad \varepsilon_{1}=\left[\begin{array}{ll}
\varepsilon_{11}^{T} & \varepsilon_{12}^{T}
\end{array}\right]^{T} \\
& \dot{\varepsilon}_{2}=A_{0} \varepsilon_{2}+\left[\begin{array}{c}
0 \\
f_{t}(l)
\end{array}\right] \quad \varepsilon_{2}=\left[\begin{array}{ll}
\varepsilon_{21}^{T} & \varepsilon_{22}^{T}
\end{array}\right]^{T} \\
& \dot{v}_{0}=A_{0} v_{0}+\left[\begin{array}{c}
0_{m \times 1} \\
\tau_{a}
\end{array}\right] \quad v_{0}=\left[\begin{array}{ll}
v_{01}^{T} & v_{02}^{T}
\end{array}\right]^{T}
\end{aligned}
$$

where $\varepsilon_{01}(t), \varepsilon_{02}(t), \varepsilon_{11}(t), \varepsilon_{12}(t), \varepsilon_{21}(t), \varepsilon_{22}(t), v_{01}(t)$, $v_{02}(t) \in \mathbb{R}^{m}$ and thus $\varepsilon_{0}(t), \varepsilon_{1}(t), \varepsilon_{2}(t), v_{0}(t) \in \mathbb{R}^{2 m}$. Based on the structure of the filters, state estimator for $r(t)$, denoted by $\hat{r}(t) \in \mathbb{R}^{2 m}$, is designed in the following manner

$$
\hat{r}=\varepsilon_{0}+A_{1} \varepsilon_{1}+A_{2} \varepsilon_{2}+\left[\begin{array}{cc}
J^{-1} & 0_{m x m} \\
0_{m x m} & J^{-1}
\end{array}\right] v_{0} .
$$

The state estimation error, denoted by $\tilde{r}(t) \in \mathbb{R}^{2 m}$, is defined as follows

$$
\tilde{r}=\left[\begin{array}{c}
\tilde{r}_{1} \\
\tilde{r}_{2}
\end{array}\right] \triangleq r-\hat{r} .
$$

where substituting (28) yields

$$
\left[\begin{array}{c}
\tilde{r}_{1} \\
\tilde{r}_{2}
\end{array}\right]=\left[\begin{array}{l}
r_{1}-\varepsilon_{01}+J^{-1}\left(B \varepsilon_{11}+R_{a} \varepsilon_{21}-v_{01}\right) \\
r_{2}-\varepsilon_{02}+J^{-1}\left(B \varepsilon_{12}+R_{a} \varepsilon_{22}-v_{02}\right)
\end{array}\right] .
$$

We can now obtain $\dot{\theta}(t)$ by computing $r_{2}(t)$ from (30) and substituting in (23) as

$$
\dot{\theta}=\tilde{r}_{2}+\varepsilon_{02}-J^{-1} B\left(\varepsilon_{12}+\theta\right)-J^{-1} R_{a} \varepsilon_{22}+J^{-1} v_{02} .
$$

To analyze the stability of the filter, we take the time derivative of (29) and obtain the following estimation error dynamics

$$
\dot{\tilde{r}}=\dot{r}-\dot{\varepsilon}_{0}-A_{1} \dot{\varepsilon}_{1}-A_{2} \dot{\varepsilon}_{2}+\left[\begin{array}{cc}
J^{-1} & 0_{m x m} \\
0_{m x m} & J^{-1}
\end{array}\right] \dot{v}_{0}
$$

where the time derivative of (28) was utilized. Substituting (25) and the set of linear filters in (27) into (32), it is easy to obtain

$$
\dot{\tilde{r}}=A_{0} \tilde{r}
$$


From the robot link control law given by (13), it is clear that we need to investigate the stability of $\eta_{f}(t)$ as well. Taking the time derivative of (11) yields

$$
\begin{aligned}
\dot{\eta}_{f}= & \frac{\partial f_{t}(l)}{\partial l}\left[J_{j}(q) \dot{q}+R_{a} \dot{\theta}\right] \\
& -\left[J_{j}^{T}(q)\right]^{+}\left\{-\dot{Y}_{d} \hat{\phi}_{r}-Y_{d} \dot{\hat{\phi}}_{r}+k K_{s} \dot{e}_{f}-K_{s} \dot{e}\right\} \\
& -\frac{d}{d t}\left[J_{j}^{T}(q)\right]^{+}\left\{-Y_{d} \hat{\phi}_{r}+k K_{s} e_{f}-K_{s} e\right\} .
\end{aligned}
$$

In the last line of the above expression, from the time derivative of the pseudo inverse of the Jacobian matrix, the time derivative of joint position vector and thus the auxiliary error signal $\eta(t)$ shows up. In view of this, the last line can be rewritten as

$$
\begin{aligned}
& \frac{d}{d t}\left\{\left[J_{j}^{T}(q)\right]^{+}\right\}\left\{-Y_{d} \hat{\phi}_{r}+k K_{s} e_{f}-K_{s} e\right\} \\
= & \Psi_{1}\left(e, e_{f}, t\right)+\Psi_{2}\left(e, e_{f}, t\right) \eta
\end{aligned}
$$

where $\Psi_{1}\left(e, e_{f}, t\right) \in \mathbb{R}^{m}$ and $\Psi_{2}\left(e, e_{f}, t\right) \in \mathbb{R}^{m \times m}$ are known and available functions. After pre-multiplying both sides of (34) with $J$ and then making use of (5), (6), (14), (31), (35), we can obtain

$$
\begin{aligned}
J \dot{\eta}_{f}= & W_{1} \phi_{1}-J \Omega_{1} \eta+J \frac{\partial f_{t}(l)}{\partial l} R_{a} \tilde{r}_{2} \\
& +\frac{\partial f_{t}(l)}{\partial l} R_{a}\left(v_{02}-R_{a} \varepsilon_{22}\right)
\end{aligned}
$$

where $W_{1}\left(e, e_{f}, l, \varepsilon_{02}, \varepsilon_{12}, \hat{\phi}, t\right) \in \mathbb{R}^{m \times p_{1}}$ is a known and available regressor matrix and $\phi_{1} \in \mathbb{R}^{p_{1}}$ is an unknown constant parameter vector, and both are obtained from

$$
\begin{aligned}
W_{1} \phi_{1}= & J \frac{\partial f_{t}(l)}{\partial l} J_{j}(q)\left(\dot{q}_{d}+\alpha_{1} e+\alpha_{2} e_{f}\right) \\
+ & J \frac{\partial f_{t}(l)}{\partial l} R_{a}\left(\varepsilon_{02}-J^{-1} B\left(\varepsilon_{12}+\theta\right)\right) \\
-J\left[J_{j}^{T}(q)\right]^{+} & \left\{-\dot{Y}_{d} \hat{\phi}+k K_{s}\left(-\alpha_{3} e_{f}+\alpha_{2} e\right)\right. \\
& \left.-K_{s}\left(-\alpha_{1} e-\alpha_{2} e_{f}\right)\right\}-J \Psi_{1} .
\end{aligned}
$$

In (36), the auxiliary matrix $\Omega_{1}\left(e, e_{f}, t\right) \in \mathbb{R}^{m \times m}$, which includes known and measurable terms, is defined as

$\Omega_{1} \triangleq \frac{\partial f_{t}(l)}{\partial l} J_{j}(q)-\left[J_{j}^{T}(q)\right]^{+}\left(Y_{d} \Gamma Y_{d}^{T}+k^{2} K_{s}+K_{s}\right)+\Psi_{2}$.

In order to backstep one more time, we define a new auxiliary error signal, denoted by $\eta_{L}(t) \in \mathbb{R}^{m}$, as

$$
\eta_{L}(t) \triangleq v_{02}-u_{L}
$$

where $u_{L}(t) \in \mathbb{R}^{m}$ is an auxiliary input like term. Utilizing (39), and adding and subtracting $\frac{\partial f_{t}(l)}{\partial l} R_{a} u_{L}(t)$ to the right-hand side of (36) results in

$$
\begin{aligned}
J \dot{\eta}_{f}= & W_{1} \phi_{1}+J \Omega_{1} \eta+J \frac{\partial f_{t}(l)}{\partial l} R_{a} \tilde{r}_{2} \\
& +\frac{\partial f_{t}(l)}{\partial l} R_{a}\left(u_{L}+\eta_{L}-R_{a} \varepsilon_{22}\right) .
\end{aligned}
$$

From the subsequent stability analysis, we design the auxiliary input $u_{L}(t)$ in the following manner

$$
u_{L}=\Lambda\left(-K_{f} \eta_{f}-W_{1} \hat{\phi}_{1}+\frac{\partial f_{t}(l)}{\partial l} R_{a}^{2} \varepsilon_{22}\right)
$$

where the auxiliary variable $\Lambda(l) \in \mathbb{R}^{m \times m}$ is defined as

$$
\Lambda \triangleq\left[\frac{\partial f_{t}(l)}{\partial l} R_{a}\right]^{-1}
$$

and $K_{f} \in \mathbb{R}$ is a control gain designed as

$$
\begin{aligned}
K_{f}= & k_{f}+k_{n 2} \bar{J}^{2}\left\|\Omega_{1}\right\|_{i \infty}^{2}+k_{n 3} \bar{J}^{2}\|\Lambda\|_{i \infty}^{2} \\
& +k_{n 4}\left\|J_{j}^{T}(q)\right\|_{i \infty}^{2}
\end{aligned}
$$

where $k_{f} \in \mathbb{R}$ is a constant control gain, $k_{n 2}, k_{n 3}, k_{n 4} \in \mathbb{R}$ are constant scalar nonlinear damping gains, and $\bar{J}$ is the upper bound of actuators inertia matrix. In (41), $\hat{\phi}_{1}(t) \in \mathbb{R}^{p_{1}}$ is the estimate of the unknown parameter vector $\phi_{1}$, and is updated according to

$$
\dot{\hat{\phi}}_{1}=\Gamma_{1} W_{1}^{T} \eta_{f}
$$

with $\Gamma_{1} \in \mathbb{R}^{p_{1} \times p_{1}}$ being a positive definite, constant, diagonal, adaptation gain matrix. After substituting (41) into (40), closed-loop dynamics for $\eta_{f}(t)$ can be obtained as

$J \dot{\eta}_{f}=-K_{f} \eta_{f}+W_{1} \tilde{\phi}_{1}+J \Omega_{1} \eta+J \frac{\partial f_{t}(l)}{\partial l} R_{a} \tilde{r}_{2}+\frac{\partial f_{t}(l)}{\partial l} R_{a} \eta_{L}$

where $\tilde{\phi}_{1}(t) \in \mathbb{R}^{p_{1}}$ is the parameter estimation error vector and is defined as

$$
\tilde{\phi}_{1} \triangleq \phi_{1}-\hat{\phi}_{1}
$$

We continue the control design by investigating the dynamics of $\eta_{L}(t)$. We take the time derivative of (39), and then substitute (3), (5), (14), (27), (31), (36), and after some straightforward mathematical manipulations, we obtain

$$
\dot{\eta}_{L}=\Omega_{2}+W_{2} \phi_{2}+\Omega_{3} \eta+\Omega_{4} \tilde{r}_{2}+\tau_{a}
$$

where $W_{2}(\cdot) \in \mathbb{R}^{m \times p_{2}}$ is an available regressor matrix, $\phi_{2} \in \mathbb{R}^{p_{2}}$ includes unknown system parameters, $\Omega_{2}(\cdot) \in$ $\mathbb{R}^{m}, \Omega_{3}(\cdot) \in \mathbb{R}^{m \times n}, \Omega_{4}(\cdot) \in \mathbb{R}^{m \times m}$ are auxiliary terms that include known and available signals. According to the subsequent stability analysis, we design the actuator control input as follows

$$
\tau_{a}=-K_{L} \eta_{L}-\Omega_{2}-W_{2} \hat{\phi}_{2}-\left[\frac{\partial f_{t}(l)}{\partial l} R_{a}\right]^{T} \eta_{f}
$$

with $K_{L} \in \mathbb{R}$ being a control gain designed as

$$
K_{L}=k_{L}+k_{n 5}\left\|\Omega_{3}\right\|_{i \infty}^{2}+k_{n 6}\left\|\Omega_{4}\right\|_{i \infty}^{2}
$$

where $k_{L} \in \mathbb{R}$ is a constant control gain, $k_{n 5}, k_{n 6} \in \mathbb{R}$ are constant scalar nonlinear damping gains, and $\hat{\phi}_{2}(t) \in \mathbb{R}^{p_{2}}$ is the estimate of the uncertain parameter vector $\phi_{2}$ which is designed to be updated as follows

$$
\dot{\hat{\phi}}_{2}=\Gamma_{2} W_{2}^{T} \eta_{L}
$$


with $\Gamma_{2} \in \mathbb{R}^{p_{2} \times p_{2}}$ being a positive definite, constant, diagonal, adaptation gain matrix. After substituting (48) into (47), the closed-loop dynamics for $\eta_{L}(t)$ can be obtained as

$$
\dot{\eta}_{L}=-K_{L} \eta_{L}+W_{2} \tilde{\phi}_{2}+\Omega_{3} \eta+\Omega_{4} \tilde{r}_{2}-\left[\frac{\partial f_{t}(l)}{\partial l} R_{a}\right]^{T} \eta_{f}
$$

where $\tilde{\phi}_{2}(t) \in \mathbb{R}^{p_{2}}$ is the parameter estimation error vector and defined as

$$
\tilde{\phi}_{2} \triangleq \phi_{2}-\hat{\phi}_{2}
$$

\section{Stability ANALYSIS}

In this section, the stability of the closed-loop system will be investigated by utilizing Lyapunov-based arguments.

Theorem 1: For the tendon driven robot manipulator system given by dynamic equations (1)-(3), link position tracking adaptive controller given by (13), (41), (48), and adaptation laws given by (14), (44), (50), in conjunction with actuator velocity error generating filter given by (27) and (28), semi-global asymptotic link position tracking is guaranteed in the sense that

$$
\|e(t)\| \rightarrow 0 \text { as } t \rightarrow \infty
$$

provided the nonlinear damping gains are selected to satisfy the following conditions

$$
\begin{aligned}
\min \left\{\gamma, 1, k_{f}, k_{L}\right\} & >\sum_{i=2}^{6} \frac{1}{4 k_{n i}} \\
k_{n 1} & >\frac{\lambda_{2}}{\lambda_{1}}\|s(0)\|^{2}+1
\end{aligned}
$$

where $\gamma \in \mathbb{R}$ is some positive bounding constant satisfying $\min \left\{\alpha_{2}, \alpha_{1} K_{s}, \alpha_{3} K_{s}\right\}-\frac{1}{4 \alpha_{2} k_{n 1}}>\gamma>0$, and $s(t) \in$ $\mathbb{R}^{(3 n+p) \times 1}, \lambda_{1}, \lambda_{2} \in \mathbb{R}$ are defined as follows

$$
\begin{aligned}
& s \triangleq\left[\begin{array}{llll}
e^{T} & e_{f}^{T} & \eta^{T} & \tilde{\phi}_{r}^{T}
\end{array}\right]^{T} \\
& \lambda_{1} \triangleq \frac{1}{2} \min \left\{m_{1}, \lambda_{\min }\left(K_{s}\right), \lambda_{\min }\left(\Gamma^{-1}\right)\right\} \\
& \lambda_{2} \triangleq \frac{1}{2} \max \left\{m_{2}, \lambda_{\max }\left(K_{s}\right), \lambda_{\max }\left(\Gamma^{-1}\right)\right\}
\end{aligned}
$$

where $\lambda_{\min }(\cdot)$ denotes the minimum eigenvalue of a matrix.

Proof: The proof is conducted in four sub-parts. At the beginning of the proof, we present the partial stability analysis for robot dynamics given by (1). The results of this analysis will afterwards be used in the composite stability analysis of the overall closed-loop system.

We define a non-negative Lyapunov-like function, denoted by $V_{1}\left(\eta, e, e_{f}, \tilde{\phi}_{r}\right) \in \mathbb{R}$, as follows

$$
V_{1} \triangleq \frac{1}{2} \eta^{T} M(q) \eta+\frac{1}{2} e^{T} K_{s} e+\frac{1}{2} e_{f}^{T} K_{s} e_{f}+\frac{1}{2} \tilde{\phi}_{r}^{T} \Gamma^{-1} \tilde{\phi}_{r}
$$

which can be bounded from below and above as

$$
\lambda_{1}\|x\|^{2} \leq \lambda_{1}\|s\|^{2} \leq V_{1} \leq \lambda_{2}\|s\|^{2} .
$$

Taking the time derivative of (59), then substituting (5), (6), (14), (16), (45), (51), and then cancelling common terms, we obtain

$$
\begin{aligned}
\dot{V}_{1}= & \eta^{T}\left[-\alpha_{2} k M(q) \eta+\chi\right]-\alpha_{1} K_{s}\|e\|^{2} \\
& -\alpha_{3} K_{s}\left\|e_{f}\right\|^{2}+\eta^{T} J_{j}^{T}(q) \eta_{f} .
\end{aligned}
$$

Using the definition of the control gain $k$ in (15), and the upper bounds of $M(q)$ and $\chi(t)$ in (8), we can reach the following upper bound for the right-hand side of (61)

$$
\begin{aligned}
\dot{V}_{1} \leq & -\alpha_{2}\|\eta\|^{2}-\alpha_{1} K_{s}\|e\|^{2}-\alpha_{3} K_{s}\left\|e_{f}\right\|^{2} \\
& +\eta^{T} J_{j}^{T}(q) \eta_{f}+\frac{1}{4 \alpha_{2} k_{n 1}}\|x\|^{2}
\end{aligned}
$$

where the following nonlinear damping argument [20] was also utilized

$$
-\alpha_{2} k_{n 1} \rho^{2}(\|x\|)\|\eta\|^{2}+\|\eta\| \rho(\|x\|)\|x\| \leq \frac{1}{4 \alpha_{2} k_{n 1}}\|x\|^{2} .
$$

Using the definition of $x(t)$ in (10), another upper bound can be reached as

$$
\dot{V}_{1} \leq-\gamma\|x\|^{2}+\eta^{T} J_{j}^{T}(q) \eta_{f}
$$

where $\gamma$ was introduced in (54).

As the second step, we present the stability analysis for the actuator velocity error generating filters. We define a nonnegative scalar function, denoted by $V_{2}(\tilde{r}) \in \mathbb{R}$, as

$$
V_{2} \triangleq \tilde{r}^{T} P_{0} \tilde{r}
$$

where $P_{0} \in R^{2 m \times 2 m}$ is a positive definite, symmetric, constant matrix chosen to satisfy

$$
A_{0}^{T} P_{0}+P_{0} A_{0}=-I_{2 m} .
$$

After taking the time derivative of (65), we obtain

$$
\dot{V}_{2}=\dot{\tilde{r}}^{T} P_{0} \tilde{r}+\tilde{r}^{T} P_{0} \dot{\tilde{r}}
$$

and substituting (33) and (66) yields

$$
\dot{V}_{2}=-\tilde{r}^{T} \tilde{r}=-\left\|\tilde{r}_{1}\right\|^{2}-\left\|\tilde{r}_{2}\right\|^{2} .
$$

As the third step, we define a non-negative scalar function, denoted by $V_{3}\left(\eta_{f}, \eta_{L}, \tilde{\phi}_{1}, \tilde{\phi}_{2}\right) \in \mathbb{R}$, as

$$
V_{3} \triangleq \frac{1}{2} \eta_{f}^{T} J \eta_{f}+\frac{1}{2} \eta_{L}^{T} \eta_{L}+\frac{1}{2} \tilde{\phi}_{1}^{T} \Gamma_{1}^{-1} \tilde{\phi}_{1}+\frac{1}{2} \tilde{\phi}_{2}^{T} \Gamma_{2}^{-1} \tilde{\phi}_{2} .
$$

Taking the time derivative of (69), substituting (44), (45), (50), (51), using the control gains $K_{f}$ in (43) and $K_{L}$ in (49), an upper bound for $\dot{V}_{3}(t)$ can be obtained as

$$
\begin{aligned}
\dot{V}_{3} \leq & -k_{f}\left\|\eta_{f}\right\|^{2}-k_{L}\left\|\eta_{L}\right\|^{2}+\frac{\|\eta\|^{2}}{4 k_{n 2}}+\frac{\left\|\tilde{r}_{2}\right\|^{2}}{4 k_{n 3}} \\
& -k_{n 4}\left\|J_{j}^{T}(q)\right\|_{i \infty}^{2}\left\|\eta_{f}\right\|^{2}+\frac{\|\eta\|^{2}}{4 k_{n 5}}+\frac{\left\|\tilde{r}_{2}\right\|^{2}}{4 k_{n 6}} . \text { (70) }
\end{aligned}
$$

Finally, in order to investigate the closed-loop system stability, we construct a non-negative scalar function, denoted by $V(t) \in \mathbb{R}$, by summing (59), (65), (69), as

$$
V \triangleq V_{1}+V_{2}+V_{3}
$$


After taking the time derivative of (71), substituting (64), (68) and (70), and then simplifying the resulting expression, we obtain the following expression

$$
\begin{aligned}
\dot{V} \leq & -\gamma\|x\|^{2}-\left\|\tilde{r}_{1}\right\|^{2}-\left\|\tilde{r}_{2}\right\|^{2}-k_{f}\left\|\eta_{f}\right\|^{2}-k_{L}\left\|\eta_{L}\right\|^{2} \\
& +\frac{\|\eta\|^{2}}{4 k_{n 2}}+\frac{\left\|\tilde{r}_{2}\right\|^{2}}{4 k_{n 3}}+\frac{\|\eta\|^{2}}{4 k_{n 5}}+\frac{\left\|\tilde{r}_{2}\right\|^{2}}{4 k_{n 6}} \\
& +\eta^{T} J_{j}^{T}(q) \eta_{f}-k_{n 4}\left\|J_{j}^{T}(q)\right\|_{i \infty}^{2}\left\|\eta_{f}\right\|^{2} .
\end{aligned}
$$

Applying a similar nonlinear damping argument to the one in (63) to the last line of (72), the upper bound for $\dot{V}(t)$ can be written as

$$
\begin{aligned}
\dot{V} \leq & -\gamma\|x\|^{2}-\left\|\tilde{r}_{1}\right\|^{2}-\left\|\tilde{r}_{2}\right\|^{2}-k_{f}\left\|\eta_{f}\right\|^{2}-k_{L}\left\|\eta_{L}\right\|^{2} \\
& +\frac{\|\eta\|^{2}}{4 k_{n 2}}+\frac{\left\|\tilde{r}_{2}\right\|^{2}}{4 k_{n 3}}+\frac{\|\eta\|^{2}}{4 k_{n 4}}+\frac{\left\|\tilde{r}_{2}\right\|^{2}}{4 k_{n 5}}+\frac{\left\|\tilde{r}_{2}\right\|^{2}}{4 k_{n 6}} .
\end{aligned}
$$

The expression given in (73) can further be upper bounded as follows

$$
\dot{V} \leq-\left(\min \left\{\gamma, 1, k_{f}, k_{L}\right\}-\sum_{i=2}^{6} \frac{1}{4 k_{n i}}\right)\|z\|^{2}
$$

where $z(t) \in R^{(3 n+4 m) \times 1}$ is defined as follows

$$
z \triangleq\left[\begin{array}{lllll}
x^{T} & \tilde{r}_{1}^{T} & \tilde{r}_{2}^{T} & \eta_{f}^{T} & \eta_{L}^{T}
\end{array}\right]^{T} .
$$

Provided the gain condition in (54) is satisfied, the upper bound for $\dot{V}(t)$ in (74) can be reformulated as

$$
\dot{V} \leq-\delta\|z\|^{2}
$$

for some positive bounding constant $\delta \in \mathbb{R}$.

Standard signal chasing arguments can now be utilized to demonstrate boundedness of all the signals under the closedloop operation. Barbalat's Lemma [18] can then be utilized to prove that $z(t)$ is asymptotically stable, and thus it is easy to see that the tracking objective is met in the sense that (53) is satisfied.

\section{CONCLUDING REMARKS}

In this work, we have presented a partial state feedback adaptive controller for tendon driven robot manipulators subject to parametric uncertainty in the system dynamics. Despite the lack of exact knowledge of the system parameters and lack of velocity measurements of both links and actuators, the proposed controller ensured trajectory tracking in the sense that, the link position tracking error signal is forced to go to zero. Stability of the closed-loop system and boundedness of system states are proven via Lyapunov based arguments.

\section{REFERENCES}

[1] S. C. Jacobsen, H. Ko, E. K. Iverson, and C. C. Davis, "Antagonistic control of a tendon driven manipulator," in Proc. of IEEE Int. Conf. on Robotics and Automation, 1989, pp. 1334-1339.

[2] M. Kaneko, W. Paetsch, and H. Tolle, "Input-dependent stability of joint torque control of tendon-driven robot hands," IEEE Tr. on Industrial Electronics, vol. 39, no. 2, pp. 96-104, 1992.

[3] M. T. Mason and J. K. S. Jr., Robot Hands and the Mechanics of Manipulation. Cambridge, MA, USA: MIT Press, 1985.
[4] S. Ma, S. Hirose, and H. Yashinada, "Design and experiments for a coupled tendon-driven manipulator," IEEE Control Systems Magazine, vol. 13, no. 1, pp. 30-36, 1993.

[5] H. Kobayashi and R. Ozawa, "Adaptive neural network control of tendon driven mechanisms with elastic tendons," Automatica, vol. 39, pp. 1509-1519, 2003.

[6] S. Chang, J. Lee, and H. Yen, "Kinematic and compliance analysis for tendon driven robotic mechanisms with flexible tendons," Mechanism and Machine Theory, vol. 40, pp. 728-739, 2005.

[7] S. C. Jacobsen, J. E. Wood, D. F. Knutti, and K. B. Biggers, "The utah/m.i.t. dextrous hand: Work in progress," Int. J. of Robotics Research, vol. 3, no. 4, pp. 21-50, 1984.

[8] G. Palli, "Model and control of tendon actuated robots," Ph.D. dissertation, Ph.D. dissertation, DEIS, University of Bologna, Bologna, Italy, 2007.

[9] J. J. Lee, "Tendon-driven manipulators: Analysis, synthesis and control," Ph.D. dissertation, Ph.D. Dissertation, Ph.D. 91-2 University of Maryland, College Park, MD, USA, 1991.

[10] T. Nakayama and H. Fujimoto, "Trajectory tracking control of tendon driven robots using delayed force feedback," in Proc. of Int. Conf. on Mechatronics, Kumamoto, Japan, 2007.

[11] K. Haiya, S. Komada, and J. Hirai, "Control of tendon-driven robotic mechanism by nonlinear springs and hysteresis characteristics," in Proc. of IEEE Int. Conf. on Mechatronics, Malaga, Spain, 2009.

[12] — - "Tension control of tendon mechanisms by compensation of nonlinear spring characteristic equation error," in IEEE Workshop on Advanced Motion Control, Nagaoka, Japan, 2010, pp. 42-47.

[13] T. Wimbock, C. Ott, and G. Hirzinger, "Immersion and invariance control for an antagonistic joint with nonlinear mechanical stiffness," in Proc. IEEE Int. Conf. Decision and Control, Atlanta, GA, USA, $1128-1135$, p. 2010.

[14] S. Sastry and M. Bodson, Adaptive control: Stability, Convergence and Robustness. Eaglewood Cliffs, N.J., USA: Prentice Hall Co., 1989.

[15] M. Krstic, I. Kanellakopoulos, and P. Kokotovic, Nonlinear and Adaptive Control Design. New York, NY, USA: John Wiley \& Sons, 1995.

[16] S. Y. Lim, D. Dawson, and K. Anderson, "Re-examining the nicosiatomei robot observer-controller from a backstepping perspective," Control Systems Technology, IEEE Transactions on, vol. 4, no. 3, pp. 304-310, May 1996.

[17] P. Kokotovic, "The joy of feedback: nonlinear and adaptive," Control Systems, IEEE, vol. 12, no. 3, pp. 7-17, June 1992. 